\title{
Klimakterium virile
}

\section{Wann profitiert Mann vom Hormonersatz?}

\begin{abstract}
Leistungseinbußen, Libidoverlust, Depression und Gewichtszunahme beim älteren Mann können Symptome eines Testosteronmangels sein.
\end{abstract}

— „Voraussetzung für die Diagnose eines Androgendefizits ist der Nachweis einer eindeutigen klinischen Symptomatik eines Testosteronmangels", betonte Dr. Ulrich Deuß, Köln. Dazu gehören neben der Libidoreduktion und der erektilen Dysfunktion eine verminderte Muskelmasse bzw. -kraft, ein erhöhter Körperfettanteil, eine verminderte Knochendichte bzw. Osteoporose oder eine verminderte Vitalität bzw. eine Depression. Mit standardisierten Fragebögen könne die Symptomatik zuverlässig erfasst werden. Aufgrund der geringen Spezifität dieser Symptome reichen sie für die Diagnose eines Hypogonadismus allein jedoch nicht aus.

\section{Testosteron morgens messen!}

Um die Diagnose „Hypogonadismus“ zu sichern, ist die wiederholte Messung des morgendlichen Testosteronspiegels unverzichtbar. Für die Normgrenzen des Testosterons liegen zwar bisher keine allgemein akzeptierten Werte vor.
„Doch Patienten mit einem Testosteronwert über $350 \mathrm{ng} / \mathrm{dl}(12 \mathrm{nmol} / \mathrm{l})$ bedürfen sicher keiner Substitution", betonte Deuß. Nur Patienten mit einem Testosteron < $230 \mathrm{ng} / \mathrm{l}(8 \mathrm{nmol} / \mathrm{l})$ profitierten in aller Regel von einer Substitution. Liegen die Werten dazwischen, ist eine weitergehende Diagnostik, evtl. die Bestimmung des freien Testosteros oder des bioverfügbaren Testosterons, sinnvoll.

Bei Nachweis eines Testosteronmangels muss eine weitere differenzialdiagnostische Abklärung der Ursache erfolgen. Dabei gilt es, insbesondere zwischen einem primären und einem sekundären Hypogonadismus zu unterscheiden. Dies erfordert die Bestimmung des LH/FSH und des Prolaktins.

\section{Wirkung bereits nach drei Monaten}

Die Indikation für eine Testosteronsubstitution besteht grundsätzlich nur bei entsprechender Symptomatik. Ziel der Substitution müsse deshalb neben einer Verbesserung bzw. des Erhalts der sekundären Geschlechtsmerkmale insbesondere eine Verbesserung der Sexualfunktion und des physischen und psychischen Wohlbefindens sein, so
Deuß. Aber auch eine Optimierung der Körperzusammensetzung mit Verbesserung der Muskelmasse und der Muskelkraft, Rückgang der Fettmasse sowie Erhalt bzw. Verbesserung der Knochendichte im Sinne einer Osteoporoseprophylaxe ist ein wichtiges Therapieziel. „Bis auf die Veränderungen am Knochen können die Effekte einer Testosteronsubstitution bereits nach drei bis sechs Monaten gut beurteilt werden", so Deuß. Wenn die gewünschte Besserung nicht einsetze, müsse die Indikation nochmals kritisch hinterfragt und ggf. die Therapie beendet werden.

\section{Kontraindikationen für die Testosteronsubstitution}

Kontraindikationen für eine Testosteronsubstitution sind Mamma- und Prostatakarzinom. Aber auch ein unklarer Prostatabefund, eine Erhöhung des PSA-Wertes über $3 \mathrm{ng} / \mathrm{ml}$ ohne weitere urologische Abklärung, eine Erythrozytose mit einem Hämatokrit über 50\%, ein Hyperviskositätssyndrom, eine unbehandelte Schlafapnoe und eine Herzinsuffizienz NYHA III oder IV verbieten eine Testosteronsubstitution.

STI

\section{Testosteronsubstitution}

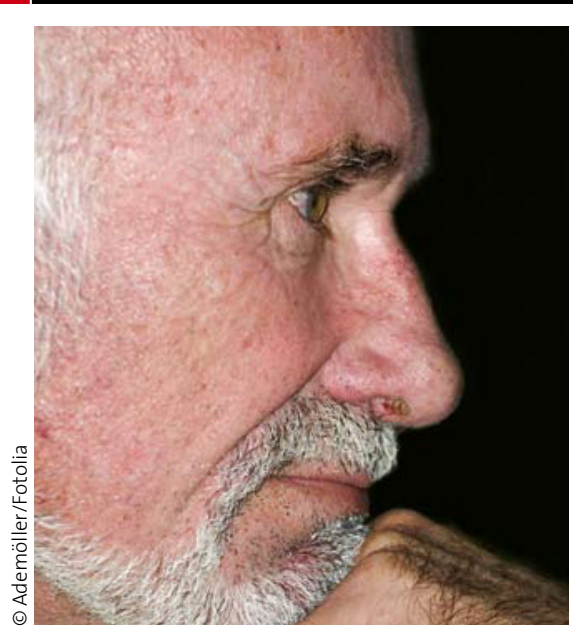

Bei Testosteronsubstitution regelmäßig Blutbild, PSA-Wert und Lipide kontrollieren!

\section{Oral, intramuskulär oder transkutan?}

Im Allgemeinen wird Testosteron in Form eines intramuskulären Depotpräparates appliziert, und zwar in einer Dosierung von $250 \mathrm{mg}$ Testosteronenantat, das in Abständen von zwei bis drei Wochen gegeben wird. Eine Alternative ist die Gabe von $1000 \mathrm{mg}$ Testosteronundecanoat alle drei Monate. Aber auch verschiedene Testo-sterongele sowie ein -pflaster stehen heute zur transdermalen Applikation zur Verfügung. Weil mögliche unerwünschte Ereignisse während der Behandlung (insbesondere erhöhte Hk-Werte) ein schnelles Absetzen der Testosteronsubstitution erfordert, sollte man kurz wirksame Präparate gegenüber lang wirksamen Depotpräparaten zu Beginn einer Therapie bevorzugen. Nicht ausreichend wirksam und daher nicht empfehlenswert ist die orale Gabe von Testosteron.

Eine Testosteronsubstitution erfordert regelmäßige klinische und laborchemische Verlaufskontrollen. Dabei sollte der Testosteronspiegel im unteren bis mittleren Normbereich liegen. Darüber hinaus sind regelmäßige Kontrollen des Blutbildes, des PSA-Wertes und des Lipidstatus erforderlich, und zwar zunächst in dreimonatigen, später in einjährigen Abständen. Kontrollen der Knochendichte sind erst nach einer Substitutionsdauer von zwei Jahren sinnvoll. 